\title{
Evaluación continua de la labor docente universitaria
}

\author{
Alejandra Consejo ${ }^{\mathrm{a}, \mathrm{b}}$ y Pascual Sevillano ${ }^{\mathrm{c}}$ \\ a'Departamento de Física Aplicada, Escuela de Ingeniería y Arquitectura, Universidad de Zaragoza, \\ alejandra.consejo@unizar.es; ${ }^{b}$ Institute of Physical Chemistry, Polish Academy of Sciences (Varsovia, Polonia); \\ 'Departamento de Física Aplicada, Facultad de Ciencias, Universidad de Zaragoza, psevi@unizar.es
}

\section{\$EWWDW}

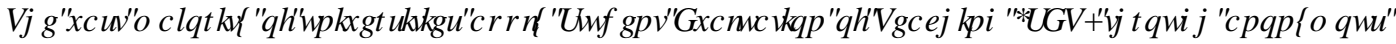

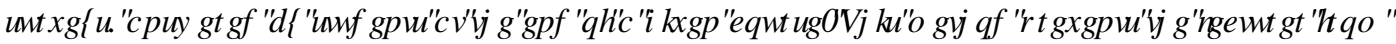

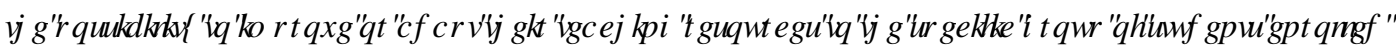

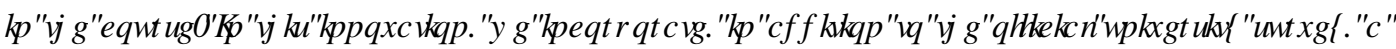

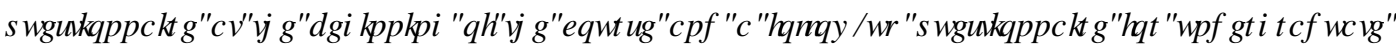

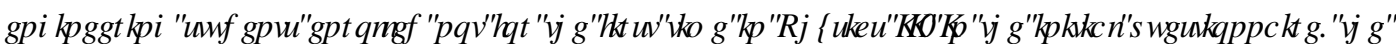

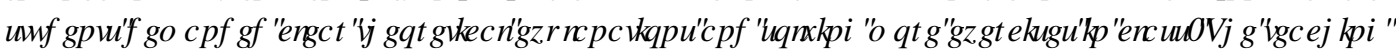

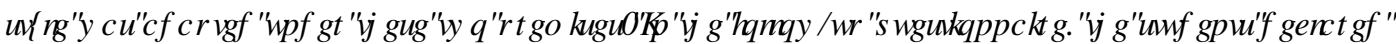

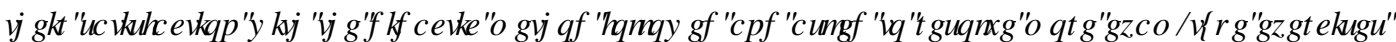

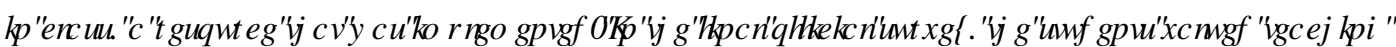

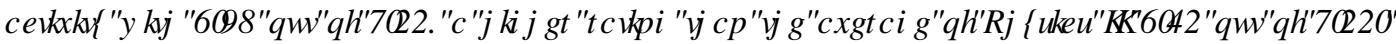

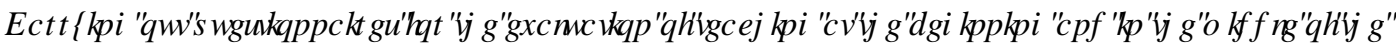

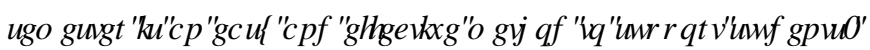

\section{. HZRLGVUUDFKID

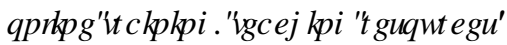

(]

\section{HXP HQ}

La gran mayoría de universidades evalúan la actividad docente por medio de encuestas anónimas, respondidas por los estudiantes al finalizar la docencia de una determinada asignatura. Este método no da margen de actuación al docente para mejorar o adaptar sus recursos didácticos al grupo concreto de alumnos matriculados en la asignatura. En esta innovación incorporamos, además de la encuesta oficial de la universidad, un cuestionario al comienzo del curso y un cuestionario de seguimiento para los alumnos de diversas ingenierías matriculados no por primera vez en la asignatura Física II. En el cuestionario inicial los estudiantes demandaron explicaciones teóricas claras y la resolución de más ejercicios en clase. La docencia de la asignatura se adaptó bajo estas dos premisas. En el cuestionario de seguimiento los estudiantes declararon su agrado con el método didáctico y pidieron que se realizasen más ejercicios tipo examen en clase, recurso que se implementó. En la encuesta final oficial los estudiantes valoraron la actividad docente con un 4.76 sobre 5.00, calificación más alta que la media de la asignatura 4.20 sobre 5.00. Realizar cuestionarios para la evaluación de la docencia a principio y a mitad de cuatrimestre es un método de fácil implantación y efectivo para apoyar a los estudiantes.

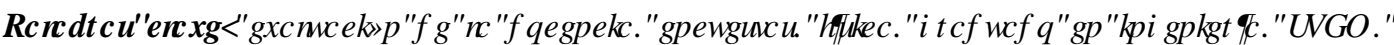
IRLP DFIY QRQQQRDUFXURVGG FUFRV 


\section{Introducción}

Hoy en día la inmensa mayoría de universidades, tanto públicas como privadas, independientemente de las áreas de conocimiento impartidas, cuentan con un sistema institucional de evaluación de la docencia (Hadad et al., 2020; Moreno-Murcia et al., 2015; Tejedor, 2012). A lo largo del tiempo, la evaluación de la docencia se ha ido desarrollando con objetivos diferentes y diversos entre los que se incluyen: conocer las demandas de los estudiantes, ayudar al profesorado a mejorar, atender a necesidades administrativas asociadas a complementos salariales de los docentes, responder al interés de la sociedad por mejorar la educación superior y rendir cuentas por parte de las universidades, apoyándose en el método de evaluación basado en resultados tangibles (Montoya et al., 2014).

La gran mayoría de las universidades se basa en el uso de los cuestionarios de los estudiantes para la evaluación docente (Gong, 2016). Dichos cuestionarios son respondidos por los estudiantes de forma anónima una vez terminada la docencia de cada asignatura. Los estudiantes deben evaluar la labor docente del profesor, así como el proceso de aprendizaje. De manera general, los cuestionarios constan de un número de afirmaciones y los estudiantes deben indicar su grado de acuerdo o desacuerdo con cada una de las sentencias propuestas (Ching, 2018). Desde hace varios años ya, y como consecuencia de la digitalización universitaria, es también habitual que los estudiantes tengan un espacio en blanco donde expresarse de forma libre sobre el docente o la asignatura. Se muestra un ejemplo de encuesta de evaluación de la actividad docente en la Figura 1. En esta encuesta ejemplo, de la Universidad de Zaragoza (UNIZAR), el alumno debe seleccionar un número de 0 (totalmente en desacuerdo) a 5 (totalmente de acuerdo) para cada una de las afirmaciones propuestas.

\begin{tabular}{l}
$\overline{\overline{i i}}$ Universidad Zaragoza \\
1. Informa de los objetivos, contenidos, bibliografia y materiales recomendados \\
2. Informa de las pruebas y criterios de evaluación que se seguirá \\
3. Informa de los fines y horario de las tutorias \\
\hline BLOQUE:A.- SOBRE LA INFORMACIÓN FACILITADA POR ESTE PROFESOR AL \\
\hline 4. Asiste a sus clases y, en caso contrario, se justifica y se sustituye o recupera \\
5. Es puntual al comenzar y al finalizar la actividad docente \\
6. EI profesor atiende las tutorias \\
BLOQUE:B.- SOBRE EL CUMPLIMIENTO DE OBLIGACIONES DE ESTE PROFESOR \\
7. Es correcto y respetuoso con el estudiante \\
8. Tiene una actitud receptiva y muestra disposición para el diálogo \\
9. Promueve el interés por la materia \\
10. Durante las tutorias ayuda a la comprensión y estudio de la asignatura \\
BLOQUE:C.- SOBRE LAS RELACIONES DE ESTE PROFESOR CON EL ESTUDIANTE \\
11. Explica de manera clara y ordenada, destacando los aspectos más importantes \\
12. Relaciona unos temas con otros de la materia \\
13. Relaciona los conceptos de la materia con sus aplicaciones \\
14. La labor de este profesor hace que la asistencia a clase facilite la comprensión de la \\
15. Realiza el seguimiento y asesora sobre las actividades o trabajos
\end{tabular}

Figura 1. Encuesta de la evaluación de la actividad docente a responder por los estudiantes tras finalizar cada asignatura en la que estén matriculados en la Universidad de Zaragoza.

Diferentes autores han demostrado que a pesar de que el uso de la evaluación de la actividad docente o SET (por sus siglas en inglés 6WGHW( YDODURQRID7HDFKQJ) para recopilar información sobre el proceso de 
enseñanza-aprendizaje sea una práctica habitual, dada la naturaleza compleja de los procesos académicos, los datos resultantes de los SET son cuestionables y limitados (Tejedor, 2012; Moreno-Murcia et al., 2015). Una de las dificultades a las que las universidades se enfrentan es la escasa participación de los estudiantes que rellenan las encuestas (Kuzmanovic et al., 2013). Parece existir la creencia más o menos generalizada entre los estudiantes de que las encuestas son una pérdida de tiempo, que no solucionan sus problemas inmediatos y que su opinión no es escuchada (Wolfer y Johnson, 2003).

Entendemos la crítica del estudiantado que reclama que su opinión debería tener un efecto más temprano, y no cuando el curso ya está finalizado y ya 'no hay solución'. Por eso, en este trabajo de investigación proponemos una innovación docente consistente en preguntar a los alumnos sobre sus expectativas y opiniones sobre impartición de la docencia antes, durante y al finalizar la docencia de la asignatura.

\section{Objetivos}

El objetivo principal de esta innovación es incorporar cuestionarios anónimos de evaluación de la docencia universitaria durante todo el periodo docente de la asignatura. Los objetivos particulares se listan a continuación:

- Antes de comenzar la docencia: conocer tanto las expectativas de los estudiantes como, si procede, las dificultades que han tenido en el pasado para superar la asignatura

- A mitad de cuatrimestre: evaluar si los alumnos están satisfechos con la metodología de la asignatura y en qué medida se han cumplido sus expectativas iniciales. Utilizar la retroalimentación positiva para mejorar el método docente.

- Al finalizar la docencia: conocer la opinión última de los alumnos sobre la evaluación docente global y juzgar la utilidad de realizar diferentes encuestas a lo largo del cuatrimestre.

\section{Desarrollo de la innovación}

Las experiencias y conclusiones que se describen en este trabajo son fruto de experiencias didácticas reales, llevadas a cabo en la Escuela de Ingeniería y Arquitectura (EINA) en la Universidad de Zaragoza en el primer cuatrimestre del curso académico 2020-2021 (septiembre-enero). En dicho periodo, debido a la crisis socio sanitaria producida por la COVID-19, las clases magistrales y de problemas se celebraron online, mientras que las prácticas de laboratorio se llevaron a cabo de forma presencial, con las pertinentes medidas de seguridad.

Para realizar esta innovación docente se seleccionó un grupo heterogéneo de estudiantes, todos ellos matriculados en un grupo rotado de la asignatura de primer curso y de formación obligatoria Física II. Los grupos rotados en EINA UNIZAR son aquellos cuya docencia se imparte en cuatrimestre distinto al indicado en el plan de estudios con el objetivo de favorecer el progreso en los estudios. Para que un estudiante pueda matricularse en un grupo rotado es condicionante que previamente haya estado matriculado en la asignatura y no la haya superado con éxito. Todos los estudiantes matriculados en el grupo rotado de Física II son alumnos de ingeniería de la rama industrial, pero pertenecen a diferentes grados, entre los que se incluyen: graduado en Tecnologías industriales, graduado en Ingeniería química, graduado en Ingeniería mecánica, graduado en Ingeniería Eléctrica y graduado en Ingeniería Electrónica y Automática.

La asignatura Física II cuenta con una elevada tasa de suspensos, es habitual que muchos estudiantes tengan dificultades para superarla y deban matricularse durante varios cursos académicos. La asignatura cubre los fundamentos de física clásica que el graduado en ingeniería necesita como base de su formación. En 
concreto, electromagnetismo, ondas y óptica son los tres pilares de la asignatura. Para el desarrollo de esta innovación docente se seleccionó un grupo de estudiantes donde todos habían ya estados previamente matriculados en la asignatura, pero nunca con la docente responsable del presente curso académico.

Se realizó un total de dos cuestionarios y una encuesta oficial, todos anónimos, a los estudiantes a lo largo del cuatrimestre. Los dos cuestionarios se prepararon a través de Google Forms (https://www.google.com/forms/about/), herramienta gratuita, y se distribuyeron vía Moodle a los estudiantes, dándoles un plazo de una semana para contestar. Los encabezamientos de cada uno de los formularios se muestran en la Figura 2. El primer cuestionario se envió a los alumnos el primer día de curso y el segundo cuestionario se preparó y envió a mitad de cuatrimestre, coincidiendo con la realización del examen parcial. Las preguntas concretas pueden consultarse en las Tablas 1 y 2 . Por otra parte, la encuesta final coincidió con el final del cuatrimestre, y se trató de la encuesta oficial preparada y distribuida por UNIZAR. Esta encuesta es común para todos los estudiantes de todos los grados. En este trabajo haremos hincapié en los cuestionarios inicial y de seguimiento, ya que constituyen el núcleo de la innovación docente.

\section{Física II - grupo ROTADO curso 2020-2021 (otoño): Cuestionario inicial}

Este cuestionario es TOTALMENTE ANÓNIMO. La idea es conocer las inquietudes del alumnado, para hacer de las clases y prácticas la asignatura más útiles para los estudiantes. Gracias por contestar.

\section{Opiniones a mitad de curso sobre Física II}

Estamos a mitad de semestre, aún nos queda mucho por delante y hay tiempo de actuación. Evalúa tu satisfacción como alumno de Física II hasta la fecha. Fijate que en cada pregunta existe la opción 'otros' para que puedas añadir lo que quieras.

Figura 2. Encabezamientos (título y descripción del cuestionario) del cuestionario inicial (arriba) y del cuestionario de seguimiento (abajo) elaborado con la plataforma gratuita Google Forms y distribuido entre los alumnos matriculados en el curso vía Moodle.

\subsection{Descripción y objetivos del cuestionario inicial}

El objetivo principal del cuestionario inicial es conocer al alumnado. Es importante recordar que todos los estudiantes del grupo elegido tienen en común que ya han estado previamente matriculados en la asignatura. En estas circunstancias parece especialmente importante preguntarles qué dificultades anteriores han tenido y qué expectativas tienen del presente curso.

La totalidad de cuestiones planteadas en el cuestionario inicial aparecen en la Tabla 1. El grupo está compuesto por un total de 52 alumnos, entorno a la mitad contestaron el cuestionario, lo que se puede consideran una muestra representativa. Entre los alumnos que contestaron al cuestionario, todos ellos con edades comprendidas entre los 19 y los 22 años, hubo más alumnos (71.4\%) que alumnas (28.6 \%), coincidiendo aproximadamente con la distribución por género de los matriculados.

Los objetivos principales del cuestionario inicial se listan a continuación:

- Conocer al alumnado en su conjunto (edad, género, cantidad de alumnos presentes).

- Investigar cuál es experiencia previa de los estudiantes con la asignatura.

- Definir las dificultades previas que han encontrado los alumnos para superar la asignatura. 


\begin{tabular}{|c|c|c|}
\hline Nro. & Enunciado de la pregunta & Opciones de respuesta \\
\hline 1 & ¿Has aprobado Física I? & $\begin{array}{l}\text { - Sí } \\
\text { - No }\end{array}$ \\
\hline 2 & Sobre convocatorias anteriores de Física II... & $\begin{array}{l}\text {-Nunca me he puesto a estudiar en } \\
\text { serio. } \\
\text {-Estudié parte de la asignatura, pero } \\
\text { lo dejé por imposible a mitad de } \\
\text { cuatrimestre. } \\
\text {-He estudiado la asignatura } \\
\text { completa, pero suspendí. } \\
\text { - Otros (respuesta libre) }\end{array}$ \\
\hline 3 & ¿Cuántas convocatorias has consumido de Física II? & $\begin{array}{l}\text { - Ninguna } \\
\text { - Una } \\
\text { - Dos } \\
\text { - Más de dos }\end{array}$ \\
\hline 4 & $\begin{array}{l}\text { Aquí tienes un espacio en blanco para expresarte. Qué } \\
\text { no te gustó de cursos anteriores (o qué sí te gustó } \\
\text { especialmente de otros profesores), qué cambiarías, en } \\
\text { qué te gustaría que se hiciese más hincapié. Puedes } \\
\text { comentar sobre las clases de teoría, de problemas, las } \\
\text { prácticas, los exámenes, lo que quieras (recuerda que } \\
\text { este cuestionario es TOTALMENTE ANÓNIMO). }\end{array}$ & Respuesta libre \\
\hline
\end{tabular}

\subsection{Descripción y objetivos del cuestionario de seguimiento}

Para evaluar el grado de satisfacción de los alumnos sobre la evolución de la asignatura, se realizó un segundo cuestionario a mitad de curso justo después de la realización del primer examen parcial. Un total de 30 alumnos contestaron al cuestionario de seguimiento. Los detalles del Cuestionario de seguimiento se muestran en la Tabla 2. Es importante tener en cuenta que los alumnos contestaron al Cuestionario de seguimiento justo después de la realización del examen parcial, pero sin conocer la calificación del mismo. Los objetivos principales del Cuestionario de seguimiento se listan a continuación:

- Analizar si las necesidades que los alumnos declararon en el cuestionario inicial se están cumpliendo con éxito

- Comprobar el grado general de satisfacción del alumnado en cuanto a la docencia y la asignatura.

En el cuestionario de seguimiento se evaluaron diferentes aspectos de la asignatura. En concreto, clases magistrales de teoría, ejercicios y evaluación (Tabla 2). 


\begin{tabular}{|c|c|c|c|}
\hline Nro. & Tema & Enunciado de la pregunta & Opciones de respuesta \\
\hline 1 & Teoría & $\begin{array}{l}\text { ¿Son suficientemente claras las clases? } \\
\text { ¿Puedes seguir los contenidos? }\end{array}$ & $\begin{array}{l}\text { - Sí } \\
\text { - No } \\
\text { - Otro (respuesta libre) }\end{array}$ \\
\hline 2 & Problemas & $\begin{array}{l}\text { ¿Consideras adecuada la cantidad de } \\
\text { problemas hechos y propuestos en } \\
\text { clase? }\end{array}$ & $\begin{array}{l}\text { - Sí } \\
\text { - No } \\
\text { - Otro (respuesta libre) }\end{array}$ \\
\hline 3 & Problemas & $\begin{array}{l}\text { ¿Te parece útil poner en común los } \\
\text { ejercicios evaluables señalando los } \\
\text { fallos más comunes? }\end{array}$ & $\begin{array}{l}\text { - Sí } \\
\text { - No } \\
\text { - Otro (respuesta libre) }\end{array}$ \\
\hline 4 & $\begin{array}{l}\text { Teoría y } \\
\text { problemas }\end{array}$ & ¿Asistes a clase regularmente? & $\begin{array}{l}\text { - Sí } \\
\text { - No } \\
\text { - Otro (respuesta libre) }\end{array}$ \\
\hline 5 & $\begin{array}{l}\text { Teoría y } \\
\text { problemas }\end{array}$ & $\begin{array}{l}\text { Si no asistes a clase regularmente, } \\
\text { ¿cuál es el motivo? }\end{array}$ & Respuesta libre \\
\hline 6 & Evaluación & $\begin{array}{l}\text { Suponiendo que te has presentado al } \\
\text { examen parcial, ¿qué tal crees que te } \\
\text { ha ido? }\end{array}$ & $\begin{array}{l}\text { - Bien } \\
\text { - Mal } \\
\text { - No lo sé }\end{array}$ \\
\hline 7 & Evaluación & $\begin{array}{l}\text { Si consideras que el examen parcial te } \\
\text { ha ido mal, ¿cuál es el motivo? } \\
\text { (puedes marcar varios) }\end{array}$ & $\begin{array}{l}\text { - No he estudiado suficiente } \\
\text { - El examen era muy difícil } \\
\text { - Lo había preparado, pero me bloqueé } \\
\text { - Otro (respuesta libre) }\end{array}$ \\
\hline 8 & General & $\begin{array}{l}\text { Aquí tienes un espacio en blanco para } \\
\text { expresarte por si te gustaría añadir algo } \\
\text { más. Ideas, sugerencias, críticas. Todo } \\
\text { es bienvenido. }\end{array}$ & Respuesta libre \\
\hline
\end{tabular}

\section{Resultados}

\subsection{Cuestionario inicial}

Tal y como se muestra en la Figura 3, en cursos anteriores la mitad de los estudiantes abandonaron el estudio de la asignatura, pese a intentarlo. Solo un tercio declaró haber estudiado la asignatura rigurosamente, pero haber suspendido pese a ello. En el cuestionario (Tabla 1) también se dio la oportunidad a los estudiantes de expresar su opinión bajo la opción ' $R W R V$ en caso de que no se sintiesen identificados con ninguna de las tres opciones propuestas o que quisiesen completar con más información. Este es el caso de una estudiante que compartió: 


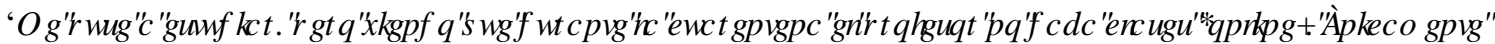

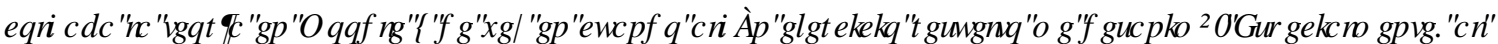

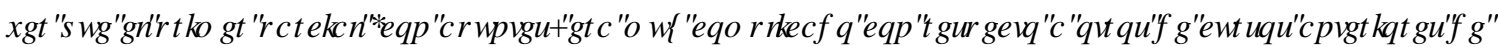

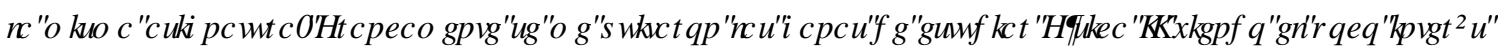

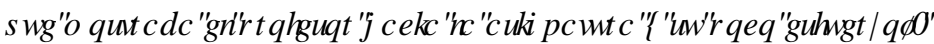

Sobre convocatorias anteriores de Física II...

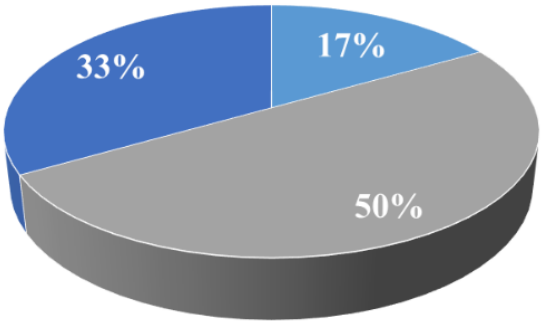

- Nunca me he puesto a estudiar en serio

" Estudié parte de la asignatura, pero lo dejé por imposible a mitad de cuatrimestre

- He estudiado la asignatura completa, pero suspendí

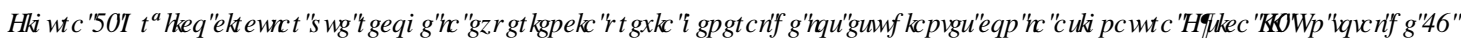
DOP QRVFRQMMDURQHOFXHMRQDURIQIFIDO

Por otra parte, aproximadamente un $40 \%$ de los estudiantes ha consumido una o ninguna convocatoria (en este último caso, implica que no se han presentado a ningún examen oficial de la asignatura), mientras que el otro $60 \%$ ha consumido ya dos o más convocatorias, tal y como indica la Figura 4.

\section{¿Cuántas convocatorias has consumido de Física II?}

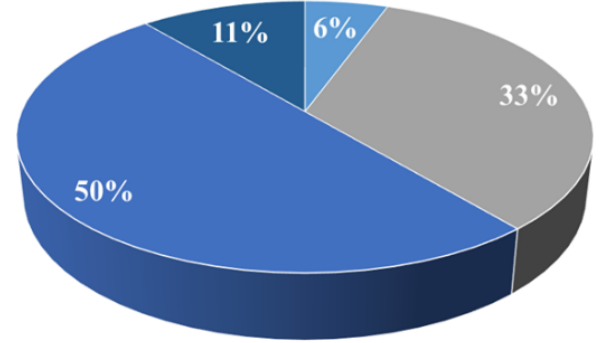

- Ninguna

$=$ Una

- Dos

- Más de dos

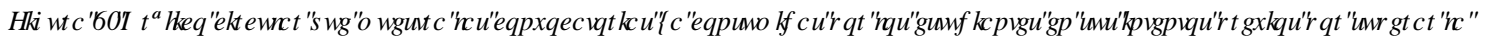

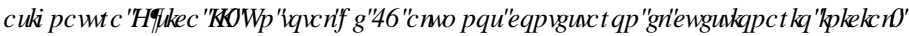

Consideramos que la pregunta más útil para mejorar el proceso docente de todo el Cuestionario inicial fue la última cuestión, donde se facilitaba un espacio en blanco para que el estudiante pudiese expresar libremente su opinión (Tabla 1). Un $85 \%$ de los estudiantes que contestaron la encuesta, escribieron algo en este apartado. En nuestra experiencia, se trata de un porcentaje de participación muy alto en una cuestión de respuesta libre. Lo interpretamos como indicativo de que los estudiantes realmente tenían algo que decir y que querían ser escuchados.

En lo siguiente, se presentan algunos de los comentarios de los estudiantes en referencia a su experiencia previa, es decir, en cursos académicos anteriores, con la asignatura:

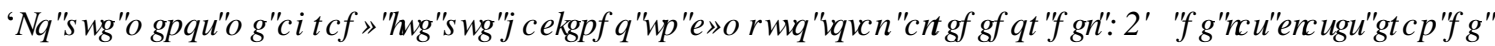

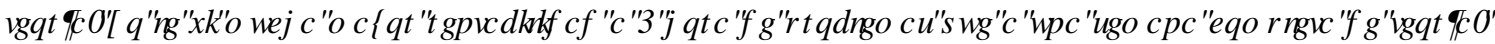

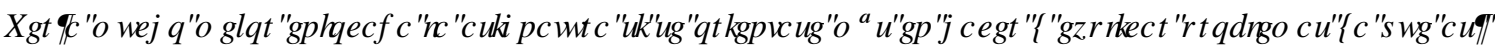

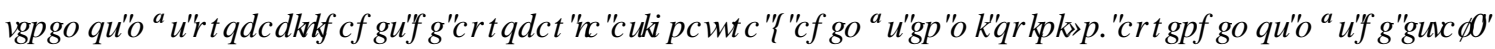

(c)) BY-NC-ND 2021, Universitat Politècnica de València 


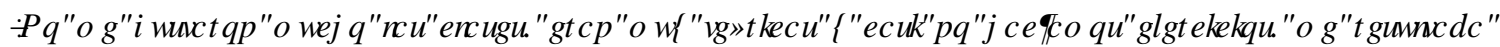
FRP SCFDQRIHQUAQGHUDOXQRVIFRQFH URVDVITI

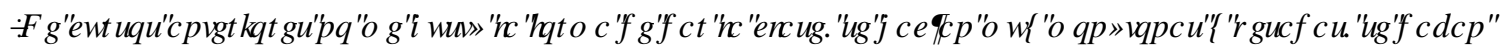

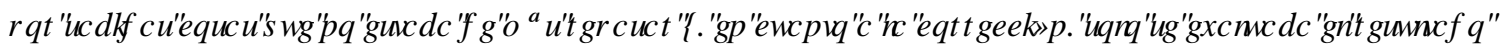

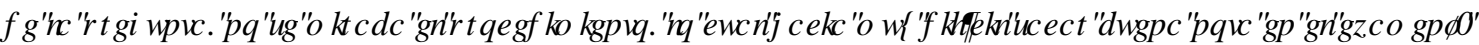

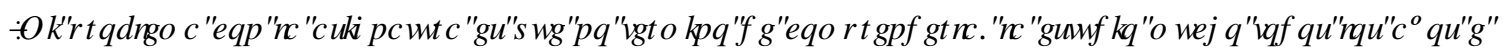

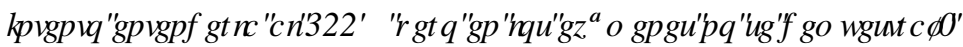

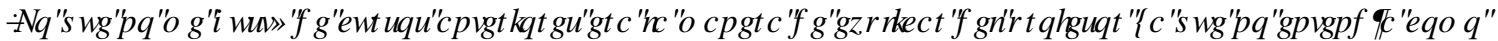

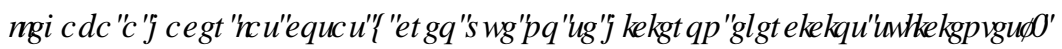

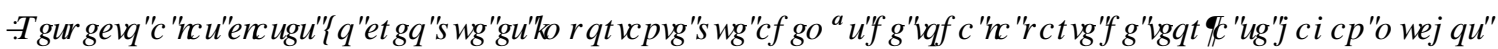

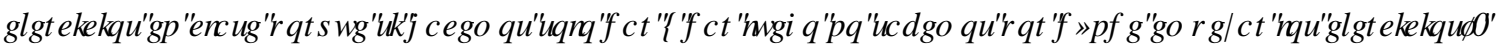

Es relevante considerar que no todos los estudiantes tuvieron en el pasado a un mismo docente. Ya que cuestionario fue anónimo para favorecer la sinceridad de los estudiantes, es complicado estimar si los alumnos que se refieren a un determinado tema que aparece de forma reiterativa, compartieron el mismo profesor en cursos anteriores o no. Aun así, se pueden extraer algunas conclusiones de la realización del cuestionario inicial que se incorporaron al método docente de la asignatura. En particular:

- Los estudiantes demandan la realización de más ejercicios prácticos.

- Las explicaciones de teoría deben ser suficientemente claras, pues parece que varios alumnos, pese a haber estudiado, no acaban de comprender la asignatura.

- Es importante que el estudiante perciba que el profesor está implicado en la asignatura.

\subsection{Cuestionario de seguimiento: Opiniones a mitad de cuatrimestre}

Teoría clara y resolución de ejercicios eran las demandas más comunes pronunciadas por los alumnos en el Cuestionario Inicial. Se intentó hacer hincapié en estos aspectos durante la evolución del curso. La totalidad de los alumnos parece estar conforme con la claridad de las explicaciones teóricas (Figura 5 izquierda), mientras que la inmensa mayoría se considera satisfecho con la cantidad de ejercicios propuestos y resueltos (Figura 5 derecha).
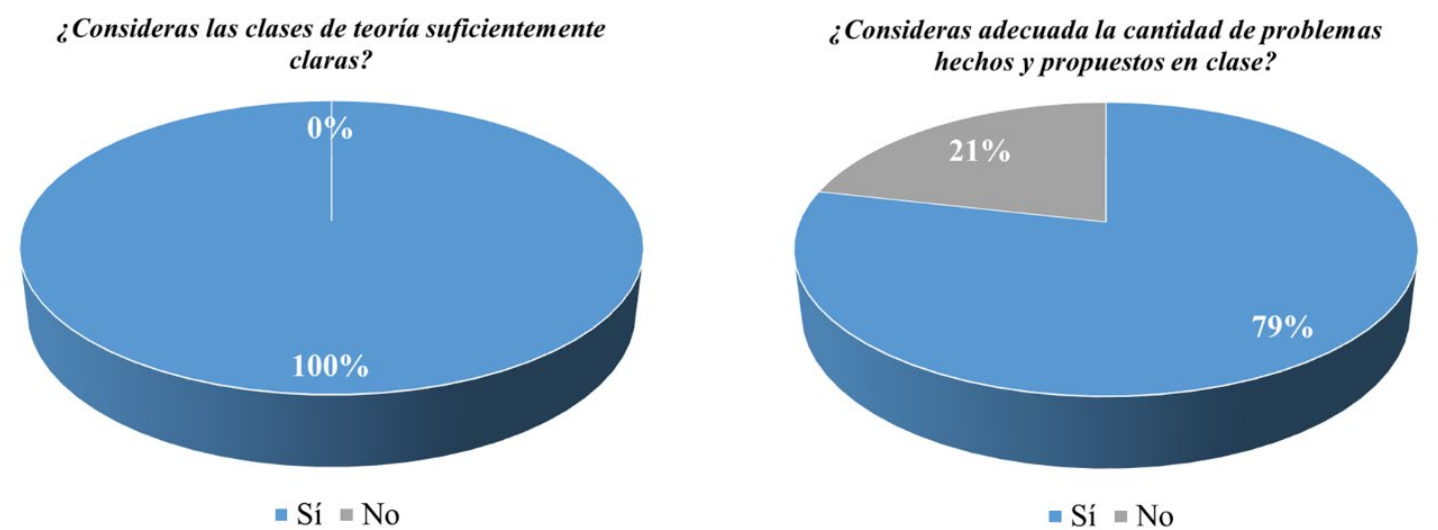

Figura 5. Gráfico circular que recoge la opinión de los estudiantes en cuanto a la claridad de las clases magistrales de teoría (izquierda) y en cuanto a la cantidad de ejercicios y problemas hechos y propuestos en clase (derecha). Un total de 30 estudiantes contestaron el cuestionario de seguimiento. 
Respecto a la asistencia a clase, un $86 \%$ de los estudiantes que respondieron a la encuesta declararon ir regularmente a clase. El resto, afirmó que les resultaba imposible atender por coincidencia horaria con otras asignaturas.

Respecto a la evaluación, la mitad de los estudiantes consideró que el primer examen parcial les había ido mal, tal y como indica la Figura 6 (contestaron este Cuestionario de seguimiento después de haber hecho el examen, pero antes de conocer la calificación del mismo). Un total de 30 alumnos contestaron al cuestionario de seguimiento, número inferior a la cantidad de alumnos que se presentaron al examen parcial. Dicho examen parcial libera temario del examen oficial final si y solo si el estudiante alcanza una calificación de 4.5 o más sobre 10. De los alumnos presentados al examen parcial (42 de los 52 matriculados), justo la mitad ( 21 alumnos) sacó un 4.5 o más sobre 10 en el examen parcial. Como el cuestionario es anónimo, es difícil asegurar que las predicciones de los alumnos fueron certeras, pero sí parece confirmase la tendencia presentada en la Figura 6.

Por otro lado, la Figura 7 analiza las principales dificultades que encontraron los estudiantes a la hora de superar el examen. En este caso, se permitió que los estudiantes marcasen tantas respuestas como como considerasen oportunas.

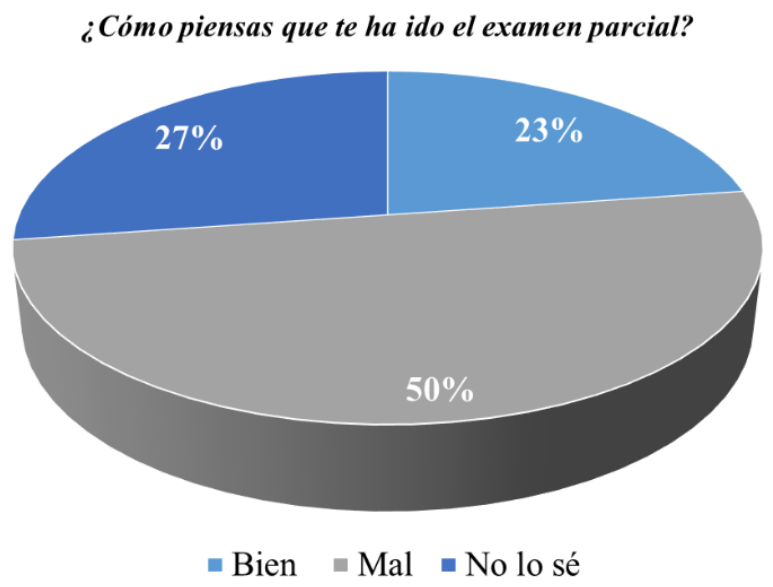

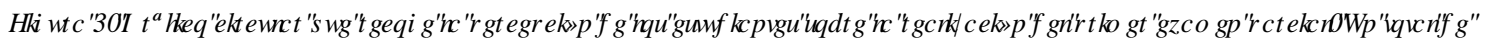

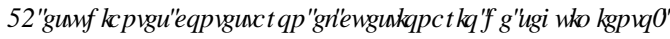

Si consideras que el examen parcial te ha ido mal, ¿cuál es el motivo?

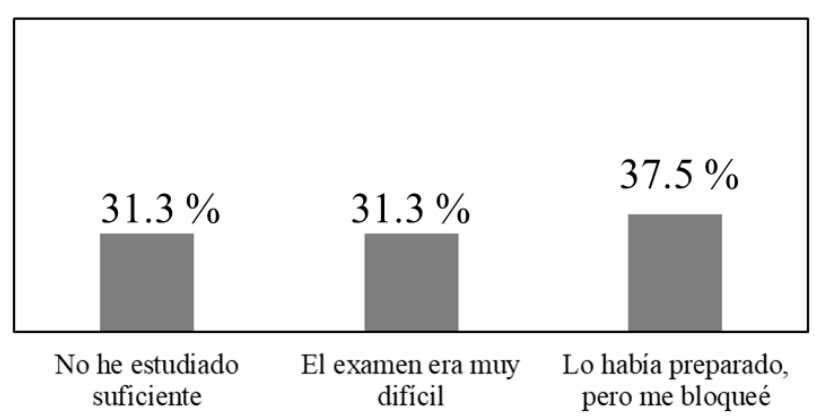

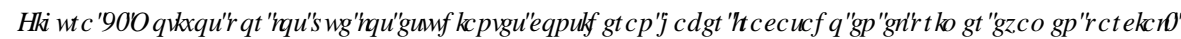

(c)) BY-NC-ND 2021, Universitat Politècnica de València

CRQJUHR, Q5 HG⿴囗十००० 
Al final del cuestionario de seguimiento se dejó un espacio libre para que los estudiantes pudiesen expresar su opinión (Tabla 2). Cabe señalar que, a diferencia de lo que ocurrió con el cuestionario inicial, menos de un tercio del total de alumnos que contestaron la encuesta de seguimiento se animaron a escribir en este apartado. En las siguientes líneas se recogen algunos de sus comentarios.

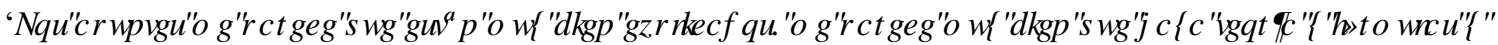

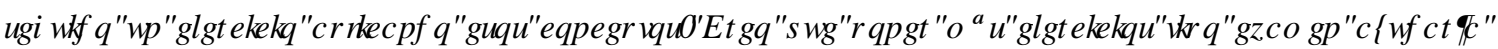
URGDYTDP i VDIIUP HRUSUSDWRRV'

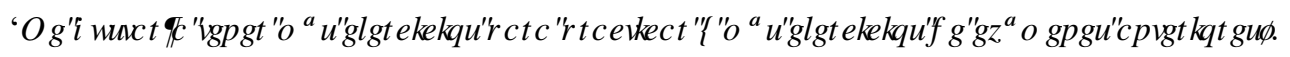

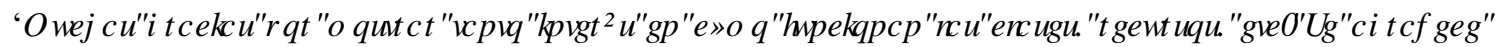

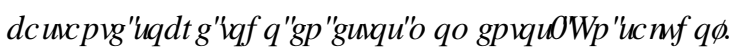

Cabe destacar que, en la realización de esta experiencia, pese al anonimato, ningún alumno fue descortés o maleducado. Todos aquellos que se mostraron más críticos con algún aspecto de la asignatura como '( $Q$ HDP HQ SDFWOP H SDUFLY $\square$ GH XQ QIYHDWSHURU DOIP SDUGR HQ FCDH lo hicieron desde el respeto. Consideramos que esto se debe, no solo a que los estudiantes sean personas educadas, sino también al hecho de que parecen agradecer este tipo de recursos, y probablemente entiendan que el profesor dejaría de implicarse y facilitar recursos semejantes si el tono de los comentarios no fuese desde el respeto.

Tras analizar el Cuestionario de seguimiento extrajimos las siguientes conclusiones:

- En general, los alumnos parecen estar contentos con la evolución del curso, consideran las explicaciones teóricas suficientemente claras y el número de ejercicios adecuado.

- Varios estudiantes han señalado que les gustaría hacer más hincapié sobre ejercicios tipo examen, es decir, ejercicios o problemas que ya han aparecido en exámenes de convocatorias anteriores.

A consecuencia de los resultados obtenidos, el método didáctico en la segunda mitad del curso apenas cambió, pero sí se añadieron más ejercicios tipo examen como habían demandado los alumnos.

\subsection{Encuesta oficial final tras finalizar la docencia}

La encuesta oficial distribuida virtualmente por UNIZAR a todos los estudiantes de todos los grados aparece en la Figura 1. Consideramos que añadir un cuestionario final no-oficial, coincidiendo con la época de preparación de exámenes, podría saturar a los alumnos. Un total de 23 estudiantes de los 52 matriculados respondieron la encuesta final oficial. Los alumnos gradaron la actividad docente, de media, con 4.76 (sobre 5.00), calificación más alta que la media de la asignatura 4.20 sobre 5.00. La encuesta oficial se realiza antes de los exámenes finales. En primera convocatoria, un $54 \%$ de los estudiantes superó la asignatura $(\mathrm{n}=28)$. De los restantes estudiantes $(\mathrm{n}=24)$, la mitad no superó el examen final $(23 \%)$ y la otra mitad no se presentó al examen (23\%). Esta tasa de abandono (23\%) es muy inferior a la del curso inmediatamente anterior (50\%, Figura 3).

\section{Conclusiones}

Realizar un cuestionario inicial y otro de seguimiento es una gran ayuda para el docente, a la hora de conocer las necesidades de los estudiantes de forma inmediata y tener la posibilidad de adaptar la docencia a dichas necesidades. Los sistemas de evaluación de docencia institucionales parecen ser poco efectivos, ya que desde que los estudiantes responden la encuesta hasta que los resultados de la misma llegan al docente suele 
pasar demasiado tiempo, la docencia de la asignatura ya ha concluido y los alumnos que contestaron la encuesta no pueden ver el retorno, en caso de que lo haya (Cadez et al., 2017; Ching et al., 2018).

En el caso de estudio aquí presentado los estudiantes tenían en común que ya habían estado anteriormente matriculados en la asignatura. En cualquier otro grupo que no cumpla esta condición tal vez la función del cuestionario inicial se desvirtúe, ya que los alumnos al no saber a qué se enfrentan, difícilmente podrán tener sugerencias de mejora. Sin embargo, si el docente conoce de antemano que la asignatura a impartir resulta complicada a los estudiantes y que posiblemente haya una cantidad significativa de alumnos que no estén matriculados por primera vez, el realizar un cuestionario inicial cobra de nuevo sentido. Por otra parte, recomendamos realizar un cuestionario de seguimiento en todos los casos, independientemente de la asignatura, del curso, o del grado. En nuestra experiencia, por una parte, resultó reconfortante saber que una de las peticiones más importantes de los alumnos (explicaciones más claras de teoría) se había visto satisfecha (Figura 5). Por otra, supimos que todavía había margen de maniobra para mejorar (resolver más ejercicios tipo examen en clase).

Los estudiantes agradecen este tipo de recursos. Valoran muy positivamente que el profesor esté pendiente de la docencia y que esté abierto a adaptarla. Por otra parte, es importante que los resultados obtenidos en los cuestionarios se comenten con los estudiantes en clase, para que sean conscientes de que el profesor considera y valora sus sugerencias, aunque haya decidido desestimarlas. En caso de que el profesor decida no adaptar la docencia a las sugerencias de los alumnos se recomienda justificar abiertamente el porqué de dicha decisión, para evitar la sensación de que el profesor ni siquiera ha evaluado las ideas propuestas y que el rellenar el cuestionario no ha sido más que una pérdida de tiempo.

Según la encuesta oficial elaborada al final del curso académico, la satisfacción general de los estudiantes subió un $11.2 \%$ respecto al curso anterior, en el que no se evaluó de forma continua la docencia. Si bien es cierto que otros aspectos pudieron ser los responsables de ese incremento, como el cambio de docente. En nuestra experiencia, el indicador que consideramos más relevante a la hora de evaluar la utilidad de la evaluación continua de la labor docente universitaria, además de los resultados de la encuesta oficial final, es la cantidad de alumnos que se presentó al examen final. La tasa de abandono (no presentados al examen final en primera convocatoria) en el grupo estudiado fue mucho menor (un $23 \%$ de matriculados) respecto al curso inmediatamente anterior, cuando un $50 \%$ de matriculados dejaron la asignatura por imposible a mitad de cuatrimestre (Figura 3).

Realizar un cuestionario inicial y/o de seguimiento no exige apenas trabajo para el docente. Google Forms cuenta con una interfaz muy intuitiva, sencilla y gratuita. Una vez escritas las preguntas y posibles respuestas, tan solo hay que generar un enlace que se debe compartir con los alumnos. Las respuestas de los alumnos se recogen en la misma interfaz de Google Forms y pueden exportarse como hoja de datos. El mismo cuestionario puede usarse para diferentes grupos o cursos académicos.

En conclusión, realizar cuestionarios no-oficiales (no institucionales) para la evaluación de la docencia a principio y a mitad de cuatrimestre es un método de fácil implantación y efectivo para adaptar la docencia a las necesidades de los estudiantes y, en consecuencia, ayudarles a aprender mejor y superar la asignatura. 


\section{Referencias}

CADEZ S, DIMOVSKI V, ZAMAN GROFF M (2017). "Research, teaching and performance evaluation in academia: the salience of quality” en 6WGHKIQ+IJKHU( GXFDURQ 2017; 42(8):1455-73.

CHING G (2018). "A literature review on the student evaluation of teaching" en + LJKHU ( GXFDMRQ ( IDOXDRQDQCE' HMHRSP HQW2018; 12(2):63-84.

GONG G (2016). "Consideration of evaluation of teaching at colleges" en 2 SHQ- RXLQDORI6RFIDOGFHQFH. 2016;4(07):82.

HADAD Y, KEREN B, NAVEH G (2020). "The relative importance of teaching evaluation criteria from the points of view of students and faculty" en \$WHMPQWQ ( YDODMRQ IQ + LJKHU ( GXFDMRQ. 2020;45(3):447-59.

KUZMANOVIC M, SAVIC G, POPOVIC M, MARTIC M (2013). "A new approach to evaluation of university teaching considering heterogeneity of students' preferences" en + IJKHU( GXFDURQ. 2013 Aug 1;66(2):153-71.

MONTOYA VARGAS J, ARBESÚ GARCÍA I, CONTRERAS PÉREZ G, CONZUELO SERRATO S (2014). "Evaluación de la docencia universitaria en México, Chile y Colombia: análisis de experiencias" en 5HIMD, EHRDP HJFDQDGH( YDODFIy Q( GXFDWDD. 2014; 7(2e), 15-42.

MORENO-MURCIA J, TORREGROSA YS, PEDREO NB (2015). "Questionnaire evaluating teaching competencies in the university environment. Evaluation of teaching competencies in the university" en

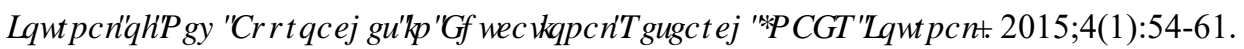

TEJEDOR FJ (2012). "Evaluación del desempeño docente" en 5HMLUD, EHRDP HUFDQD GH( IDODFIy Q ( GXFDUMD. 2012;5(1):318-27.

WOLFER TA, JOHNSON MM (2003). "Re-evaluating student evaluation of teaching: The teaching evaluation form" en - RXLQDORI 6RFIDO. RLN( GXFDURQ. 2003;39(1):111-21. 